International Journal of Advances in Materials Science and Engineering (IJAMSE) Vol.4, No.4, October 2015

\title{
EVALUATION OF MAJORITY CHARGE CARRIER AND IMPURITY CONCENTRATION USING HOT PROBE METHOD FOR MONO CRYSTALLINE SILICON (100) WAFER
}

\author{
Nahid Akter ${ }^{1}$, Sajia Afrin ${ }^{2}$, MdAbul Hossion ${ }^{3}$, Md. Alamgir Kabir ${ }^{4}$, Shirin
} Akter $^{5}$, ZahidHasan Mahmood ${ }^{6}$

\author{
1,2,3,6 Departmentof Electrical and Electronic Engineering, University of Dhaka, \\ Dhaka,Bangladesh \\ ${ }^{4}$ Department of Electrical and Electronic Engineering, Bangladesh University of Business \\ and Technology, Dhaka, Bangladesh \\ ${ }^{5}$ Accelerator Facilities Division, Atomic Energy Commission, Dhaka, Bangladesh
}

\begin{abstract}
Temperature dependent majority charge carrier concentration and impurity concentration calculations done for p-type commercial (100) silicon wafer of thickness $200 \mu \mathrm{m}$ where the wafer is heated by hot probe set up. By solving basic conductivity equation of semiconductor and Continuity \& Poisson's equation thermally generated carrier $\Delta p$ and $Q$ are found respectively for the same temperature range $50^{\circ} \mathrm{C}-80^{\circ} \mathrm{C}$ with an interval of $10^{\circ} \mathrm{C}$. For lower temperature $\Delta p$ and $Q$ values are not exactly same but for higher temperature those values agree with conventional measurement.
\end{abstract}

\section{KEYWORDS}

Hot probe, Carrier concentration, Acceptor ion concentration, Hole mobility, Diffusion Coefficient.

\section{INTRODUCTION}

Crystalline silicon exhibits some desired and predictable characteristics which lead to necessitycharacterization of the wafer during solar cell fabrication process. As instance, Evaluation of properties and charge carrier dynamic parameters such as type of semiconductor, impurity concentration, mobility of charged carrier, diffusion coefficient become of high importance in solid state electronics. Nowadays, Hall Effect technique and Mass Spectroscopy could reveal a good estimation of carrier concentration but with some considerable considerations. Therefore, hot probes technique is quite appalling for low cost endeavor. Conventional hot probe method only defines semiconductor types whereas, new hot probe technique can measure and calculate the impurity concentration and compare with the conventional measurements [1]. 


\section{Derivation of Basic Conductivity Behavior Equation in SEMICONDUCTOR}

According to the electro-neutrality rule for the initial electro-neutral state of semiconductor wafer

$n+N_{A}=p+N_{D}$

Where, $\mathrm{p}$ and $\mathrm{n}$ are majority charge carriers for p-type and n-type semiconductorrespectively. $\mathrm{N}_{\mathrm{A}}$ and $\mathrm{N}_{\mathrm{D}}$ are acceptor and donor ion concentration respectively.

From the charge conservation law, we know $p n=n_{i}^{2}$, where $n_{i}$ is the intrinsic charged carrier concentration at room temperature. Therefore, Eq. 1 can be rewritten as

$p=n+N_{A}=\frac{n_{i}^{2}}{p+N_{A}}$

Note that, for $\mathrm{p}$-type semiconductor wafer neglecting donor ion concentration $\mathrm{N}_{\mathrm{D}}$. Thesolution of Eq. (2) becomes

$$
\begin{aligned}
& \left(p-\frac{N_{A}}{2}\right)^{2}=n_{i}{ }^{2}+\left(\frac{N_{A}}{2}\right)^{2} \\
& \Rightarrow p=\frac{N_{A}}{2} \pm \sqrt{n_{i}{ }^{2}+\left(\frac{N_{A}}{2}\right)^{2}}
\end{aligned}
$$

Eq.(3) implies that, at room temperature, $p$ is the majority charge carrier for $p$-type semiconductor wafer. But at higher temperatures, this $\mathrm{p}$ values differ from $\mathrm{n}_{\mathrm{iT}}$, the intrinsic concentration at $\mathrm{T}$ temperature at Eq. (3).

There are hot and cold probes, maintained at $\mathrm{x}$ distance apart and few portions of wafer are heated, so that majority carrier concentration changes to $\mathrm{p}_{\mathrm{T}}$ due to thermally excited charge carrier density, $\Delta \mathrm{p}$. Therefore, we get

$p_{T}-p=\Delta p$

Where,

$p_{T}=\frac{N_{A}}{2} \pm \sqrt{n_{i T}{ }^{2}+\left(\frac{N_{A}}{2}\right)^{2}}$

The solution of Eq. 4 becomes

$\Delta p=\sqrt{\left(\frac{N_{A}}{2}\right)^{2}+n_{i T}{ }^{2}}-\sqrt{\left(\frac{N_{A}}{2}\right)^{2}+n_{i}{ }^{2}}$

Assume that, for the temperature variation from room temperature to $150^{\circ} \mathrm{C}$, the effective masses of the charge carriers and semiconductor bandgaps are stable. From Boltzmann relation we get

$n_{i} \propto T^{\frac{3}{2}} e^{-\frac{E g}{2 K T}}$ 
The intrinsic charged carrier concentration at room temperature becomes

$n_{i}=A T_{0}^{\frac{3}{2}} e^{-\frac{E g}{2 k T_{0}}}$

And at increased temperature it becomes

$n_{i T}=A T^{3 / 2} e^{-\frac{E g}{2 K T}}$

Where, $\mathrm{A}$ is the proportionality coefficient, $\mathrm{E}_{\mathrm{g}}$ is the band gap of a semiconductor, and $\mathrm{k}$ is the Boltzmann's constant.

DividingEq.(9) by Eq. (8) we get

$\frac{n_{i T}}{n_{i}}=R=\left(\frac{T}{T_{0}}\right)^{3 / 2} e^{\frac{E g}{2 K T_{0}}\left(1-\frac{T_{0}}{T}\right)}$

Where, $\mathrm{R}$ is the function of heating temperature.

Eq.(6) can be rewritten as

$\Delta p=\sqrt{\left(\frac{N_{A}}{2}\right)^{2}+n_{i}{ }^{2} R^{2}}-\sqrt{\left(\frac{N_{A}}{2}\right)^{2}+n_{i}{ }^{2}}$

$\Rightarrow \Delta p+\sqrt{\left(\frac{N_{A}}{2}\right)^{2}+n_{i}^{2}}=\sqrt{\left(\frac{N_{A}}{2}\right)^{2}+n_{i}^{2} R^{2}}$

By squaring both side of Eq. (11) and putting $\Delta \mathrm{p}<\mathrm{n}_{\mathrm{i}}<\mathrm{n}_{\mathrm{i}} \mathrm{R}$ we get

$\Delta p \approx \frac{n_{i}{ }^{2} R^{2}}{N_{A}}$

We can use Eq.(12) for calculating impurity concentration for (100) silicon wafer.

\section{Derivation by Using Poisson's Equation}

Thermally excited charge carriers leave the heated zone of the wafer and create a depletion region around the hot probe. Thus the diffusion equation in one-dimension form is as follows

$\int_{0}^{\infty} N(x, t) d x=Q$

Where, $\mathrm{N}(\mathrm{x}, \mathrm{t})$ is the concentration of free charged carriers at a distance $\mathrm{x}$ and time $\mathrm{t}$.

The solution of Eq. (13) by using error function gives

$$
\begin{aligned}
& N(x, t)=Q \frac{2}{\sqrt{\pi}} \int_{0}^{t} e^{\left(-\frac{x}{2 \sqrt{D t}}\right)^{2} d\left(\frac{x}{2 \sqrt{D t}}\right)} \\
& N(x, t)=Q \frac{2}{\sqrt{\pi D t}} e^{\left(\frac{x^{2}}{4 D t}\right)}
\end{aligned}
$$


Where, $\mathrm{D}$ is the diffusion coefficient of the charge carriers.

According to continuity and Poisson's equation we get

$\Delta J+\frac{\partial Q}{\partial t}=0$

$\Delta E=\frac{Q}{\varepsilon_{0} \varepsilon_{r}}$

The diffusion process described by well-known Gauss distribution using the relation $\mathrm{Q}=\mathrm{qN}(\mathrm{x}, \mathrm{t})$ in Eq.(15), we get

$\frac{\partial E}{\partial x}=\frac{q Q}{2 \pi \varepsilon_{0} \varepsilon_{r} \sqrt{\pi D t}} e^{-\frac{x^{2}}{4 D t}}$

If, $\mathrm{B}=\frac{q Q}{2 \pi \varepsilon_{0} \varepsilon_{r} \sqrt{\pi D t}}, y^{2}=\frac{x^{2}}{4 D t}$ and $\quad d x=2 \sqrt{D t d y}$

Then Eq. (16) becomes

$\frac{\partial E}{\partial x}=B e^{-y^{2}}$

For, $\mathrm{x}=\mathrm{L}=5 \mathrm{~cm}, \mathrm{t}=5 \mathrm{~s}$ and temperature $323 \mathrm{~K}-353 \mathrm{~K}, \mathrm{y}<1$, Eq. (17) can be evolved as

$\int \frac{\partial E}{\partial x}=\int B e^{-y^{2}}$

$\frac{E}{2 \sqrt{D t}}=B \int_{0}^{y} e^{-y^{2}} d y=B\left(y-\frac{y^{3}}{3}+\frac{y^{5}}{2 X 5}-\cdots\right)$

The steady state voltage, $\mathrm{V}$ measured between the hot and cold probe may be expressed from $\mathrm{E}$ by integration of the equation

$\frac{\partial V}{\partial x}=-E$

Thus, Eq. 20 may be integrated using Eq. (19) as

$\frac{V}{2 \sqrt{D t}}=-B \frac{y^{2}}{2}+B \frac{y^{4}}{4} \ldots$

$V=-2 B \sqrt{D t}\left(\frac{x^{2}}{2.4 D t}\right)=-B \frac{x^{2}}{4 \sqrt{D t}}$

$V=\frac{-q Q x^{2}}{2 \pi^{\frac{3}{2}} \varepsilon_{0} \varepsilon_{r} 4 D t}$

For, $\mathrm{x}=\mathrm{L}$, the additional thermally excited charged carriers quantity can be obtained from this equation

$Q=\frac{-V 2 \pi^{\frac{3}{2}} \varepsilon_{0} \varepsilon_{r} 4 D t}{q L^{2}}$ 
Where, negative sign indicates that the material is p-type [1] [2].

Variation of hole mobility with respect to Temperature is redrawn for $50^{\circ} \mathrm{C}-80^{\circ} \mathrm{C}$ with an interval of $10^{\circ} \mathrm{C}$ temperature shown in Fig. 1 [3].

By inserting the values of hole mobility we can calculate the diffusion coefficient using the wellknown Einstein equation

$\frac{D_{p}}{\mu_{p}}=\frac{K T}{q}$

Where, $D_{p}$ is the hole diffusion coefficient, $\mu_{\mathrm{p}}$ is the hole mobility [4].

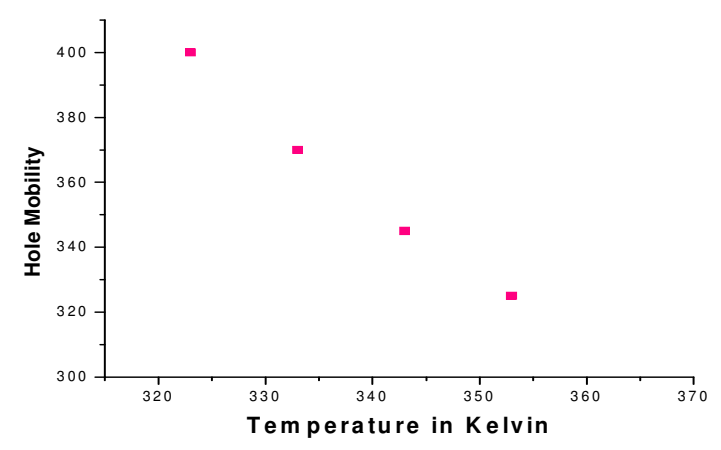

Figure 1.Temperature Vs.hole mobility for silicon.

\section{SPECification OF SiLicon WAFer}

'ReneSola' silicon wafer manufacturer provide us 6" $\times 6$ " silicon wafer with Pseudo-square (PSQ) geometry (cylinders are shaped as squares with rounded off corners). Image 1 shows some important parameters of the wafer and table 1 shows some calculated parameters.

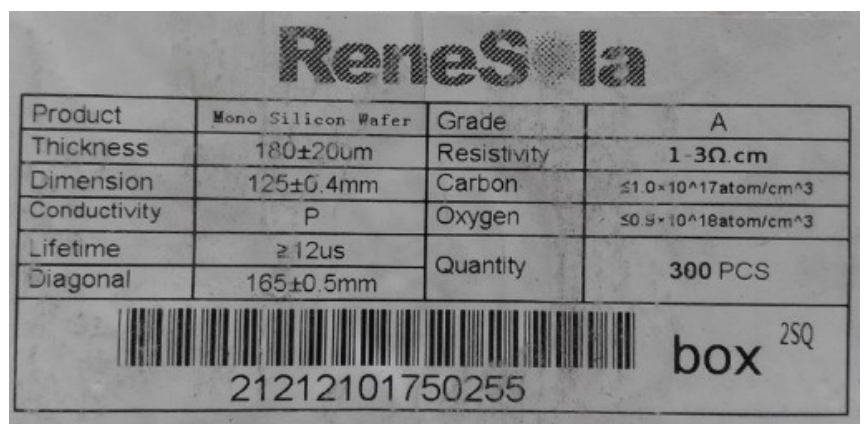

Image 1. Parameters of mono crystalline silicon wafer provided by 'ReneSola' 
Table 1. Parameters for mono silicon wafer

\begin{tabular}{|c|c|}
\hline \multicolumn{2}{|c|}{ Mono Silicon Wafer } \\
\hline Conductance type & p-type measured by hot probe setup \\
\hline Mass $(\mathrm{g})$ & $6.96 \mathrm{~g}$ measured by Microbalance \\
\hline Sheet resistivity $(\Omega /$ Square $)$ & Calculated average sheet resistivity \\
& $114.81 \Omega /$ Square by using four probe method \\
\hline Physical Resistivity $(\Omega-\mathrm{cm})$ & Calculated physical resistivity $2.3 \Omega$ - $\mathrm{cm}$ \\
\hline
\end{tabular}

\section{EXPERIMENTAL DETAILS}

A low cost indigenously made hot probe consists of a hot plate that was used to heat up the wafer and two copper probes which measures potential difference between two probes, shown in Image 2. Predefined temperature was controlled by a low cost programmable temperature controller. Some portion of a semiconductor wafer was placed on hot plate and the rest part wasplaced on cold nonconductive base. Positive probe of the meter was connected to the hot plate side wafer and negative probe of the meter was connected to the cold plate side wafer. To measure the temperature of both hot and cold probe, two k-type thermocouples were connected in contact of the hot and cold surface of the wafer.

When the wafer had begun to heat, thermally generated charge carriers hadstarted to diffuse from the hot side to cold side and createda potential difference between two probes. For various temperature profiles, these temperature gradient potential differences values had been recorded and plotted.

Negative voltage reading on the voltmeter determines that the material is p-type and the reverse is n-type. From the plotted characteristics curve, thermally excited charge carrier generation for a particular temperature can be calculated [5].

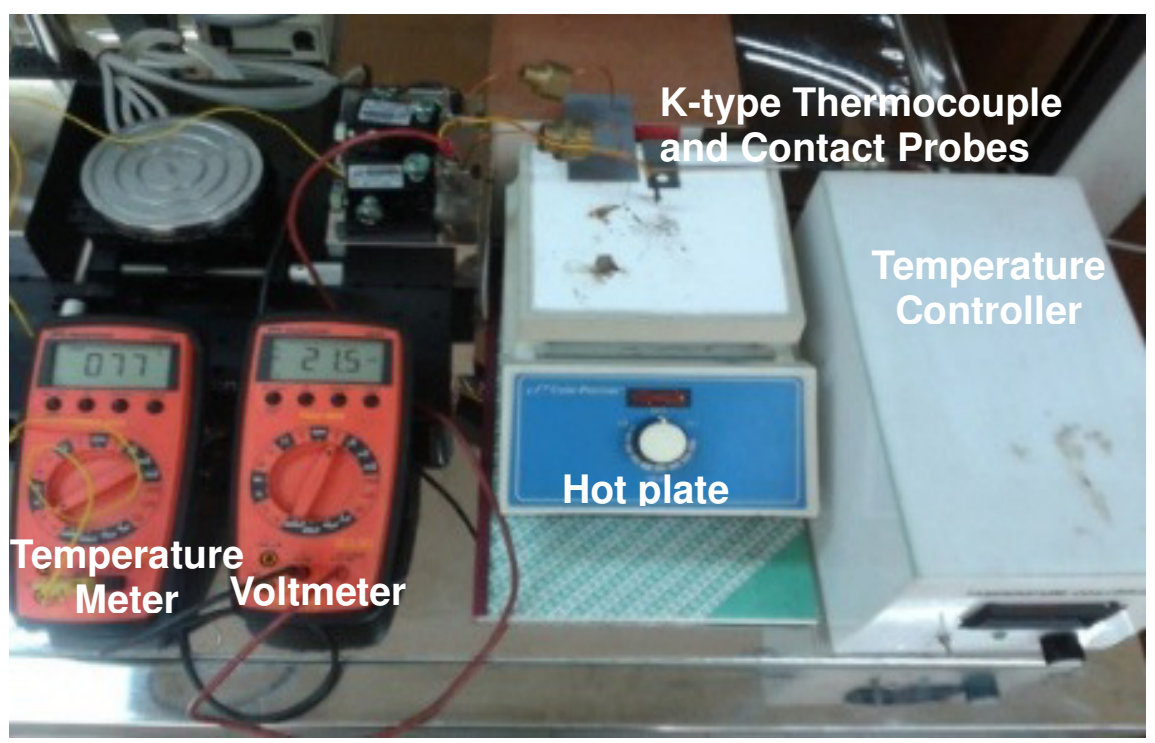

Image 2. Experimental setup of hot probe measurement 
Fig. 2 shows the graph of characteristic curves for p-type 200 micrometer thickness (100) commercial silicon wafer [5].

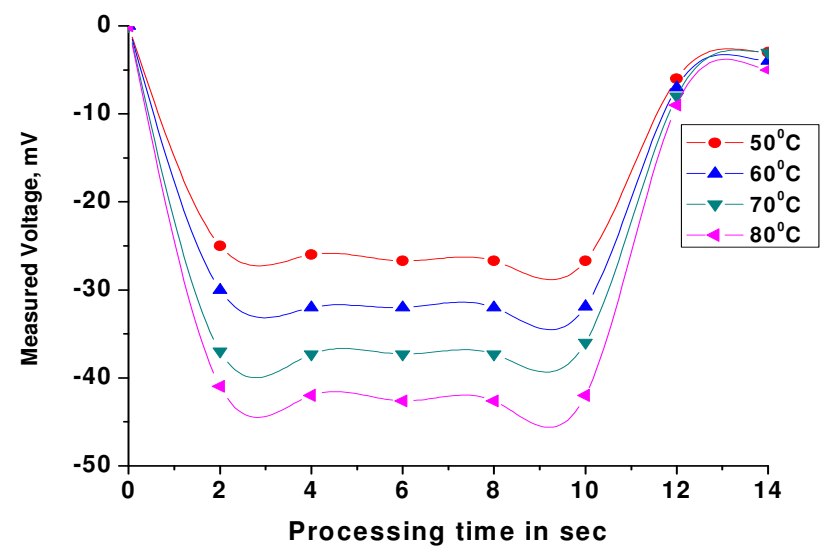

Figure 2. Characteristic curves for p-type commercial (100) silicon wafer.

The characteristic curves are divided into three-step processes:

1. Heated carriers begin to leave the heated part of the wafer surface by a diffusion mechanism. As a result, initial peak of the characteristic curve shows a built-in electric field which is developed between the electrodes.

2. Cold electrode would be simultaneously warmed up because of the diffusion of those heated carrier. So that, the built-in electric field tends to prevent the diffusion process and creates a steady state voltage. This steady state condition exists until the heated source is switched off.

3. When the hot plate is removed from the wafer then the thermally generated carriers return back to its initial states and this processis called recombination of the excited additional charged carriers.

\section{RESULTS}

\subsection{Calculated result of section 2}

Some additional data for silicon samples are

Bandgap, $E_{g}=1.12 \mathrm{eV}$, hole mobility at room temperature, $\mu_{p}=480 \mathrm{~cm}^{2} / \mathrm{V} \cdot \mathrm{s}$, intrinsic carrier concentration at room temperature, $n_{i}=1.45 \times 10^{10} / \mathrm{cm}^{3}$. Majority carrier concentration at room temperature for (100) silicon wafer becomes $p=3 \times 10^{20} / \mathrm{cm}^{3}$ by solving the Eq. (3).

Table 2.Temperature $\left({ }^{\circ} \mathrm{C}\right) \mathrm{Vs}$. hole mobility $\left(\mu_{p}\right)$, impurity concentration $\left(N_{A}\right)$, function of heating temperature $(R)$ and thermally excited charge carrier density $(\Delta p)$

\begin{tabular}{|c|c|c|c|c|}
\hline$T^{\circ} C$ & $\mu_{p}$ & $N_{A}$ & $R$ & $\Delta p$ \\
\hline 50 & 400 & $6.8 \times 10^{15}$ & 5.52 & $0.1 \times 10^{7}$ \\
\hline 60 & 370 & $7.3 \times 10^{15}$ & 10.66 & $0.4 \times 10^{7}$ \\
\hline 70 & 345 & $7.8 \times 10^{15}$ & 20.26 & $2 \times 10^{7}$ \\
\hline 80 & 325 & $8.4 \times 10^{15}$ & 36.74 & $5 \times 10^{7}$ \\
\hline
\end{tabular}




\subsection{Practical Results of section 3}

According to Eq. (24), quantity of thermally excited charge carrier could be analyzed. The steady state voltage (table 3 ) has been obtained from the characteristics curve plotted in Fig. 2 with probe distance, $\mathrm{L}=5 \mathrm{~cm}$, time interval between the initial state and steady state is 5 seconds and temperature elapses $50^{\circ} \mathrm{C}$ to $80^{\circ} \mathrm{C}$ with an interval of $10^{\circ} \mathrm{C}$.

Additional data for silicon sample is absolute permittivity, $\varepsilon_{0}=8.854 \times 10^{-12}$, relative permittivity, $\varepsilon_{r}=11.64$ and charge quanta, $q=1.6 \times 10^{-19} \mathrm{C}$. According to Eq.(25), the hole diffusion coefficient $\left(D_{p}\right)$ can be found. From Eq. (4), majority charge carrier hole could be changed due to thermal effect shown in table 2. This change increases with respect to increase of temperature.

Table 3. Temperature $\left({ }^{\circ} \mathrm{C}\right)$ Vs. steady state voltage $(m V)$, hole diffusion coefficient $\left(D_{p}\right)$ and thermally excited charge carrier $(Q)$

\begin{tabular}{|c|c|c|c|}
\hline$T^{\circ} C$ & $m V$ & $D_{p}$ & $Q$ \\
\hline 50 & 26.7 & 11.15 & $1.7 \times 10^{7}$ \\
\hline 60 & 32 & 10.63 & $1.9 \times 10^{7}$ \\
\hline 70 & 37.3 & 10.35 & $2.2 \times 10^{7}$ \\
\hline 80 & 42.64 & 9.74 & $2.3 \times 10^{7}$ \\
\hline
\end{tabular}

Table 4. Comparison between theoretical and practical data of thermally excited charge carrier values

\begin{tabular}{|c|c|c|}
\hline$T^{\circ} C$ & $\Delta p$ & $Q$ \\
\hline 50 & $0.1 \times 10^{7}$ & $1.7 \times 10^{7}$ \\
\hline 60 & $0.4 \times 10^{7}$ & $1.9 \times 10^{7}$ \\
\hline 70 & $2 \times 10^{7}$ & $2.2 \times 10^{7}$ \\
\hline 80 & $5 \times 10^{7}$ & $2.3 \times 10^{7}$ \\
\hline
\end{tabular}

\section{DISCUSSION}

As this research is linked up with previous one, a comparing discussion should be provoked. In the previous paper,wafer type (n-type or p-type) has been determined for commercial silicon wafer (thickness $200 \mu \mathrm{m}$ ) and one side polished 4 inch Si wafer (thickness $660 \mu \mathrm{m}$ )

And this measurement has been taken from hot probe characteristic curves by using low cost inhouse made hot probe measurement setup. Seebeck coefficient has also been calculated from the hot probe characteristic curves and wafer mappingthat describes the information of doping uniformity for n-type diffusion using $\mathrm{POCl}_{3}$ on p-type silicon wafer of thickness $200 \mu \mathrm{m}$ over the whole surface of wafer for both front and back sides.

On the contrary, in this paper, majority charge carrier and impurity concentration have been measured using hot probe measurement set up for the commercial silicon (Thickness:200 $\mu \mathrm{m}$ ). The experimental results are supported by solving basic conductivity equation of semiconductor and it gives the thermally generated carrier $\Delta \mathrm{p}$ values. By solving Continuity and Poisson's equation with error function and from the hot probe characteristic curves, thermally generated carrier $\mathrm{Q}$ values are obtained. For lower temperatures like at $50^{\circ} \mathrm{C}$ and $60^{\circ} \mathrm{C}, \Delta \mathrm{p}$ and $\mathrm{Q}$ values are not exactly same but at higher temperatures those values are close approximate to each other. 


\section{Conclusion}

An approach using low cost indigenously made hot probe method to estimate temperature dependent variation of majority charge carrier concentration has been experimentally investigated for silicon wafer(100). This also has given a quick measurement of the type of silicon wafer.

The critical part of this experiment was to measure the surface temperature of silicon wafer as many circumstances can contribute to faulty temperature reading. Therefore, authors have ensured good contact with the sample and the hot plate as well as from silicon wafer to thermocouple.

Possible heat loss in the system might not be an issue here as the hot probe and the thermocouple have been kept in close vicinity: also we have kept both the contacts as small as possible. In future a significant enhancement could be made to this same experiment by conducting the experiment in vacuum to avoid this kind of estimation. Another point is thermally conducting paste could be used between those contacts to improve the thermal conduction.

The measurement of thermo-generated voltage between hot and cold probe and estimation of majority charge carrier concentration agrees with value from conventional measurement. Thus the low cost indigenously made hot probe, the theoretical estimation and the results of the study are expected to serve as a useful reference for the related research endeavor.

\section{REFERENCES}

[1] Axelevitch, A., \& Golan, G. (2013), "Hot-probe method for evaluation of majority charged carriers concentration in semiconductor thin films", Factauniversitatis - series: Electronics and Energetics, vol. 26, no. 3, pp. 187-195.

[2] Golan, G. et al. (2006), "Hot-Probe Method for Evaluation of Impurities Concentration in Semiconductors." Microelectronics Journal, vo. 37, pp.910-15.

[3] Zeghbroeck, B. (2010), "Principles of semiconductor devices and heterojunctions", Upper Saddle River, N.J.: Prentice Hall.

[4] del Alamo, Jesús. 6.012 Microelectronic Devices and Circuits, Fall 2005. (MIT OpenCourseWare: Massachusetts Institute of Technology), License: Creative Commons BY-NCSA.Web:http://ocw.mit.edu/courses/electrical-engineering-and-computer-science/6-012microelectronic-devices-and-circuits-fall-2005 (Accessed onJan 1, 2015).

[5] Akter, N. et al., (2014) "Electrical Characterization and Doping Uniformity Measurement during Crystalline Silicon Solar Cell Fabrication Using Hot Probe Method." Engineering International, Journal of Asian Business Consortium, vol. 2, no. 1, pp. 38-42. 